\title{
A Small Step on the Long Road to Understanding the R Stars: CNO Cycling in Candidate R Star Progenitors
}

\author{
G. Angelou ${ }^{\mathrm{A}}$ and J. Lattanzio $^{\mathrm{A}, \mathrm{B}}$ \\ A Centre for Stellar and Planetary Astrophysics, School of Mathematical Sciences, \\ Monash University, Melbourne, VIC 3800, Australia \\ B Corresponding author. Email: john.lattanzio@sci.monash.edu.au
}

Received 2008 May 2, accepted 2008 June 9

\begin{abstract}
Recent work has proposed that a merger event between a red-giant and a He white dwarf may be responsible for the production of R stars (Izzard, Jeffery \& Lattanzio 2007). We investigate the proposed evolution and nucleosynthesis of such a model. We simulate the hypothesized late ignition of the core flash by increasing neutrino losses until ignition occurs sufficiently far from the centre that the subsequent evolution produces carbon dredge-up to the extent that the post-flash object is a carbon star. Detailed nucleosynthesis is performed within this approximation and we show that the overall properties are broadly consistent with the observations. Details will depend on the dynamics of the merger event.
\end{abstract}

Keywords: stars: evolution — stars: nucleosynthesis — R stars — He core flash

\section{Introduction}

Despite their discovery now dating back almost a century, a full explanation for the $\mathrm{R}$ stars eludes us. The division into early- $\mathrm{R}$ and later- $\mathrm{R}$ now seems to be a division into true- $\mathrm{R}$ and $\mathrm{N}$ (or $\mathrm{J}$ ), respectively. We will assume this dichotomy in what follows and pursue an explanation for the early-R stars. The main features to be explained are:

1. they are carbon stars, i.e. they have atmospheric $n(\mathrm{C})>n(\mathrm{O})$

2. their spectral type is otherwise $\mathrm{K}$;

3. they are enhanced in ${ }^{12} \mathrm{C},{ }^{13} \mathrm{C},{ }^{14} \mathrm{~N}$, but seem to have solar $[\mathrm{Fe} / \mathrm{H}]$, oxygen, and $s$-process abundances;

4. their luminosity (about $100 \mathrm{~L}_{\odot}$ ) identifies them as clump giants, that is, low mass stars burning He in their cores;

5. most peculiarly of all, long term studies by McClure (2007) failed to find any early R stars in binary systems.

Both the luminosity and solar $s$-process abundances imply the $\mathrm{R}$ stars have not reached the thermally pulsing AGB phase. In contrast are the $\mathrm{N}$ stars, rich in $s$-process elements and with luminosities in excess of $2000 \mathrm{~L}_{\odot}$, leading to their interpretation as AGB stars having undergone third dredge-up. The most thorough investigation of the composition of the R stars was that of Dominy (1984).

The fact that $\mathrm{R}$ stars are observed only as single stars leads to the notion, initially counter-intuitive, that they must all have originated as binaries. The argument is that a single star would not evolve any differently to a widely separated binary, so if $\mathrm{R}$ stars are not found in binaries at all then they cannot exist as single stars. Hence they must be exclusively binary in origin and their current singularity is assumed to be due to a merger event. Given that approximately $20 \%$ of normal late-type giants are binaries, and that none of these stars are observed in binaries, we assume that every $\mathrm{R}$ star is the product of a coalescence.

In normal low-mass single-star evolution neutrino losses at high density cause a temperature inversion in the degenerate core of stars ascending the red-giant branch. Eventually the triple- $\alpha$ reaction ignites at the point where the temperature peaks, which is no longer at the centre. A strong flash occurs, perhaps leading to $10^{9} \mathrm{~L}_{\odot}$ from $\mathrm{He}-$ burning. A convective region develops and extends from the off-centre temperature maximum almost all the way to the H-rich envelope. It seems that contact is not made between these two convective regions (except for the case of very low [Fe/H], see Fujimoto, Iben \& Hollowell 1990; Fujimoto, Ikeda \& Iben 2000; Hollowell, Iben \& Fujimoto 1990; Schlattl et al. 2001, 2002; Picardi et al. 2004; Komiya et al. 2007). After the flash dies down, there is a second flash, somewhat closer to the centre but substantially less energetic. This repeats a few times until the flash moves to the centre, and then central He burning is initiated. The energy released from the explosive Heburning has effectively lifted the degeneracy of the core and enables it to now burn He quiescently.

The first attempt at an explanation for the R stars was made by Paczyński \& Tremaine (1977). They showed that, if the core-flash could be ignited sufficiently far from the centre of the star, that is, at a much larger core mass than normal, then a dredge-up episode follows the flash and carbon is dredged to the stellar surface. This would explain the observed $n(\mathrm{C})>n(\mathrm{O})$ in the $\mathrm{R}$ stars, which are thought to be core He burning stars and, thus, would be the progeny of this unusual core flash. It remained to explain why only 
a small fraction of core flashes produced such dredge-up or, alternatively, why only a small fraction of core flashes begin at much larger core mass than normal.

This model was the preferred explanation for $\mathrm{R}$ stars until the discovery that they are all single stars. A merged binary model was the basis for a recent study by Izzard et al. (2007) to explore merger scenarios using binary star population synthesis. They identified possible formation channels that lead to an $\mathrm{R}$ star outcome. The most promising scenario was the merging of a He white dwarf and a first-ascent red giant. Typical masses of He white dwarves are about $0.15-0.2 \mathrm{M}_{\odot}$, and the red-giant mass is around $1-2 \mathrm{M}_{\odot}$. The merger is hypothesised to lead to a more rapidly rotating core than normal which then supports the core more than in the normal case. The core flash is hence delayed and ignites at a larger core mass, generating dredge-up in the manner found by Paczyński \& Tremaine (1977).

In this paper we try to take the next step in investigating this model, by looking at some basic nucleosynthetic constraints. We assume that the merger event has already occurred. Furthermore we assume that the star has returned to hydrostatic equilibrium, which allows us to use a hydrostatic stellar evolution code to model the evolution and nucleosynthesis. We try to force a core-flash event that is followed by dredge-up of carbon and see if the resulting abundances are consistent with those observed in R stars.

\section{Method}

We use the Monash version of the Mt. Stromlo stellar evolution code, MONSTAR (Frost \& Lattanzio 1996), for the evolutionary calculations. We wish to examine the nucleosynthesis that results from a core-flash that occurs unusually far from the centre of the star. The hypothesis is that this happens as a result of the merger process, possibly through spinning-up the core which delays the ignition of helium. Alternatively during the merger itself there may be ignition of the core in the very outer regions. In the absence of a code capable of calculating the merger we have resorted to a simple artifice. The usual off-centre temperature maximum seen in red-giants is due to neutrino losses in the core; since these depend mostly on the density they are higher in the centre, leading to a relative cooling compared to material at a slightly lower density just outside the very centre. We have chosen to increase these standard neutrino losses by an arbitrary factor $f_{v}$. This has the effect of cooling the core somewhat and delaying the ignition. It only affects the core, however, this is the only place where the neutrino losses are significant. We thus expect this technique to be sufficiently realistic for our purposes of forcing a more off-centre core flash.

We have evolved three models of solar composition. The first was our standard case, with $M=1.5 \mathrm{M}_{\odot}$ and $f_{v}=1$, hereinafter M1.5NL1. The second case had $f_{v}$ increased until we found that the core-flash led to a dredgeup event. This required $f_{v}=30$, so this model is referred to as M1.5NL30. We repeated this test with a $2 \mathrm{M}_{\odot}$ model and again we required $f_{v}=30$ to force a dredge-up event after the core flash. This model is hereinafter referred to as M2NL30.

The models were evolved from the main sequence through to the giant branch and the core helium flash. We followed the evolution to the beginning of quiescent core He-burning, but not beyond. This covered the dredge-up event that followed the core flash. In the following sections we compare the evolution of the regular M1.5NL1 model to M1.5NL30 to see the effect of the delayed flash and the associated dredge-up. We also compare with the slightly more massive M2NL30, which is also in the mass range for R-star progenitors predicted by Izzard et al. (2007). We then use our post-processing nucleosynthesis code MONSOON (e.g. Lattanzio et al. 1996; Lugaro et al. 2004) to investigate the resulting nucleosynthesis and how this affects the surface compositions in our proposed R stars.

Because we wish to simulate a coalesced star at a specific mass, we have ignored mass loss during the evolutionary calculations. We are well aware of the difference between our models used here and the complex events that take place during the merger of two stars. We assume that the merged object resembles a normal giant in structure, albeit with core and envelope masses that differ from those that arise during normal single-star evolution. We expect rotating cores to rapidly slow from magnetic braking. We note that, in fact, Dominy's (1984) study showed no rotational line broadening in his stars' line profiles. If merged objects are the progenitors of $\mathrm{R}$ stars, then we require their cores to still be rotating rapidly at the time of the core flash, which may mean that the mergers are required to occur only near the top of giant branch. Thus only a small fraction of the mergers are likely to then experience the dredge-up event required to make $\mathrm{R}$ stars. This is consistent with the Izzard et al. (2007) calculations, which found that if all mergers were to become R stars then they over-produced $\mathrm{R}$ stars by a factor of ten or more. Thus rotational braking is a natural way to reduce the predicted numbers to something closer to that observed.

\section{Results and Discussion}

\subsection{Structure and Evolution}

We first begin by looking at the standard evolution through the core flash (see e.g. Despain 1981; Catelan, de Freitas Pacheco \& Horvath 1996). The increasing density in the core results in growing energy loss through neutrino processes, mostly plasma emissions. This results in a relative cooling of the centre and hence an off-centre temperature maximum develops. It is at this maximum in the temperature that the He ignites, under degenerate conditions, at $m=0.20 \mathrm{M}_{\odot}$. The decoupling of temperature from the equation of state results in the thermal runaway known as the flash. This peak in the He luminosity is very large, reaching over $10^{9} \mathrm{~L}_{\odot}$ before decreasing again. Most of the energy released goes into altering the equation of state (removing the degeneracy) and the core oscillates slightly, resulting in a second, third and fourth smaller flash. These are shown in Figure 1. The star then settles down to 


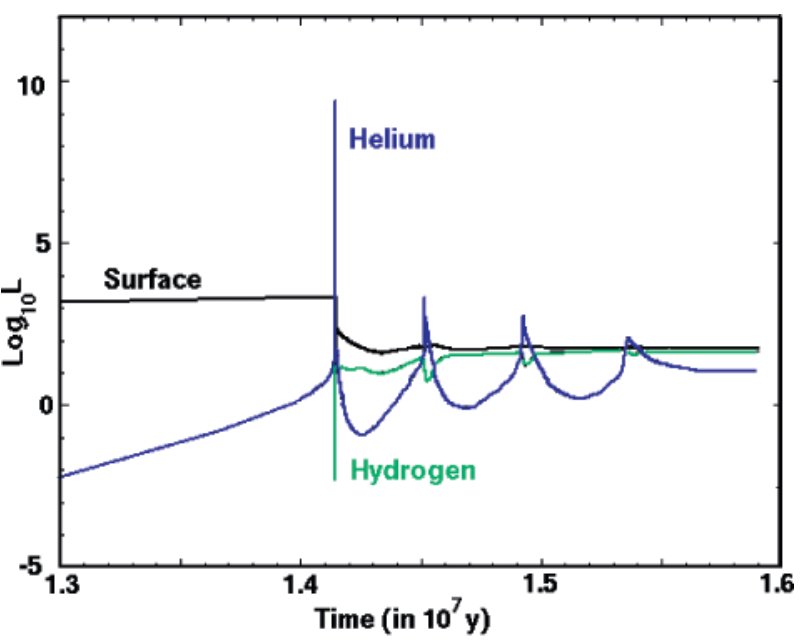

Figure 1 The variation of surface $\mathrm{H}$ and $\mathrm{He}$ luminosities during the core flash of the $M=1.5 \mathrm{M}_{\odot}$ model with normal neutrino losses. Time is measured from $t=2.67 \times 10^{9}$ years.

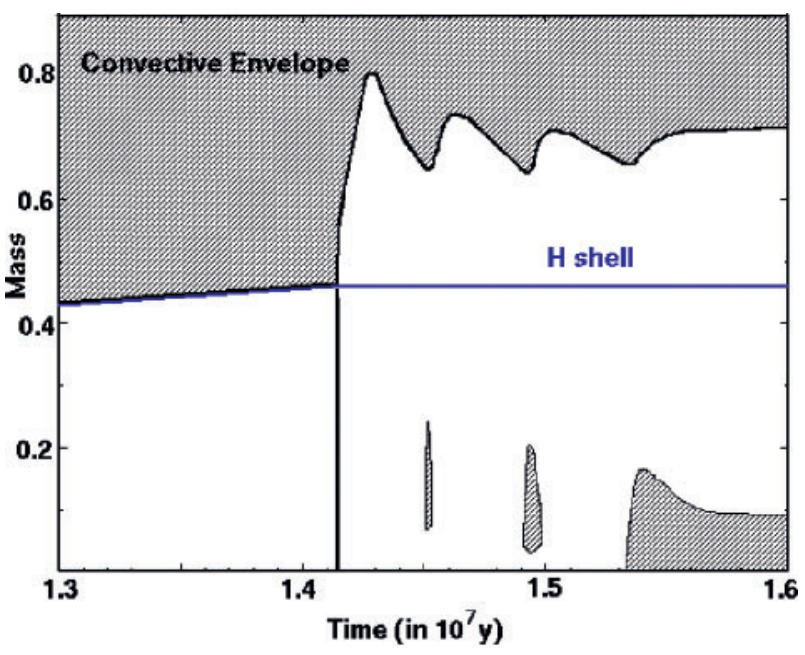

Figure 2 The variation of the convective regions during the core flash for the standard model, as shown in Figure 1.

quiescent core helium burning on the clump (for masses around $1-2 \mathrm{M}_{\odot}$ ) or the horizontal branch (for lower masses). The timescale from the major flash to quiescent He burning is found by all authors to be 1-2 million years (e.g. Despain 1981; Siess 2008), although the number of mini-flashes depends sensitively on the stellar mass and composition and quite possibly the numerical details. Figure 2 shows the extent of the convective regions during this phase of the evolution. One can see clearly the convective regions generated by the He-burning and that the fourth such flash ends in quiescent core helium burning in a convective core. This model shows typical evolution through a core flash. The initial convective pulse extends close to, but does not penetrate, the hydrogen-rich envelope. In particular, we note that there is no dredge-up of carbon following the flash.

In contrast is our model M15NL30, with neutrino losses increased by a factor of 30 . This model undergoes the

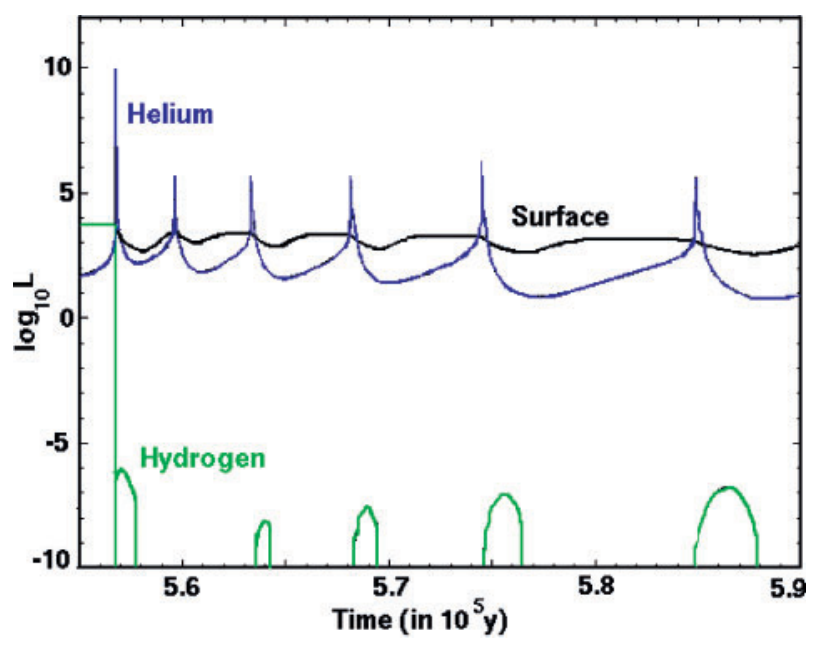

Figure 3 The variation of surface $\mathrm{H}$ and He luminosities during the core flash of the M1.5NL30 model with neutrino losses enhanced by a factor of 30 . Time is measured after $t=2.67 \times 10^{9}$ years. Note the much larger number of small He pulses, which occur on a much shorter timescale than in the standard case (Figure 1).

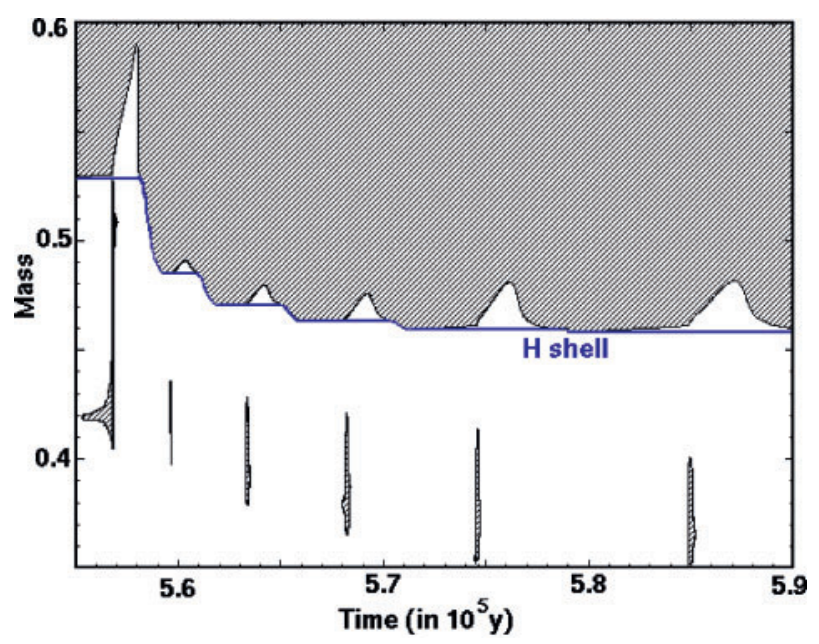

Figure 4 The variation of the convective regions during the core flash for the same model as shown in Figure 3. Note the dredge-up of material following the major pulse. This material is carbon enriched and transforms the star into a carbon star. The following smaller sub-pulses also dredge species to the envelope, but have less effect than the first event.

expected large flash but this is initiated much further out from the centre, due to the enhanced energy losses from neutrinos. In this case the ignition point is at $m=0.42 \mathrm{M}_{\odot}$, compared to $m=0.20 \mathrm{M}_{\odot}$ in the standard case. We note that this is followed by a series of many more small pulses than is seen in the standard case, and that the time between these pulses is initially very short, being of order a few thousand years. This is visible in Figure 3, and actually matches the behaviour seen in Paczyńksi and Tremaine (1977; see their Figure 2). Again, the timescale between the first pulse and the final quiescent He burning is 1-2 million years.

The convective zones in the M1.5NL30 model are shown in Figure 4. We see the substantial dredge-up resulting from the first pulse, and that there are decreasing 


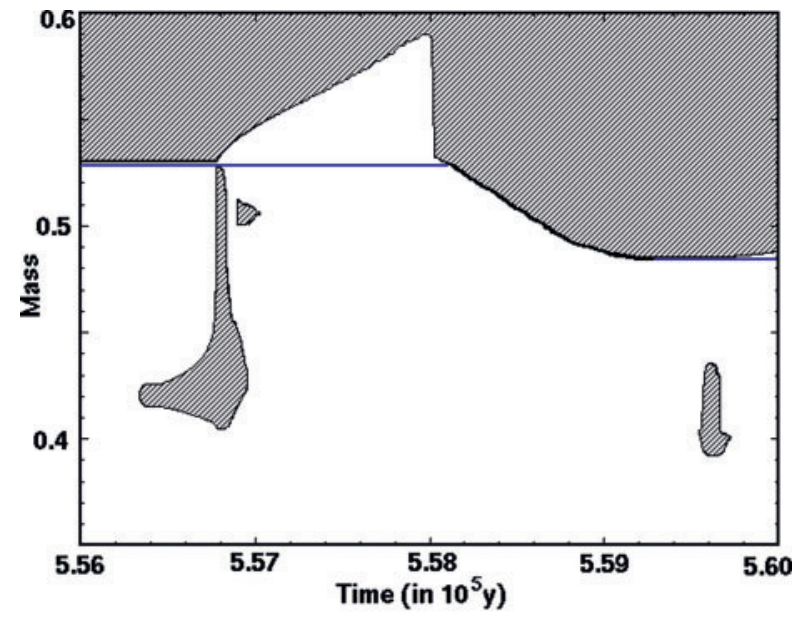

Figure 5 Close-up of the variation of the convective regions during the first major pulse in model M1.5NL30, which results in a carbon star. Once again time is measured from $t=2.67 \times 10^{9}$ years.

dredge-up events following each of the subsequent smaller pulses, also. The combined effect of these events is to reduce the hydrogen-exhausted core-mass from $0.53 \mathrm{M}_{\odot}$ to $0.46 \mathrm{M}_{\odot}$, and to enhance the surface carbon content so that the star becomes a carbon star with $n(\mathrm{C}) / n(\mathrm{O})=1.26$. A close-up of the first major dredge-up event is seen in Figure 5.

In the original calculations of the core flash there were some cases where the convective zone at the first pulse made contact with the hydrogen envelope, but these were determined as due to ignoring radiation pressure or poor numerical resolution (Despain 1981). The first (and until this work, the only) calculation to show dredge-up, much like that seen on the AGB, was Paczyńksi \& Tremaine (1977). Much like us, they 'artificially cooled' their cores to produce a delayed flash, although they do not give the details of how this was done. Our increased neutrino losses are a similar artifice. In any event, we conclude that it is the ignition of the flash much closer to the hydrogen shell that produces an expansion and subsequent behaviour, including dredge-up, that is unlike other core-flash calculations and is rather more like the dredge-up seen in AGB stars.

Indeed, without further numerical experimentation we are unable to definitively say whether the dredge-up is the result of the larger core itself or the change in the location of the ignition point (to closer to the hydrogen shell) or if both are needed. We have decided not to pursue this further at present. It is indeed possible that during a merger the rapid accretion of matter form one core onto another may trigger the flash further from the centre, and that the whole process is less tied to rotation than we have proposed. In any event, the result that we are simulating is a flash that results in dredge-up and we have succeeded in producing that, whatever the driving mechanism.

Before examining the nucleosynthesis resulting from this evolution, we also investigated the behaviour of a $2-\mathrm{M}_{\odot}$ model with identically enhanced neutrino losses. The evolution was qualitatively similar, and the convection zones during the flash are seen in Figure 6. This model also

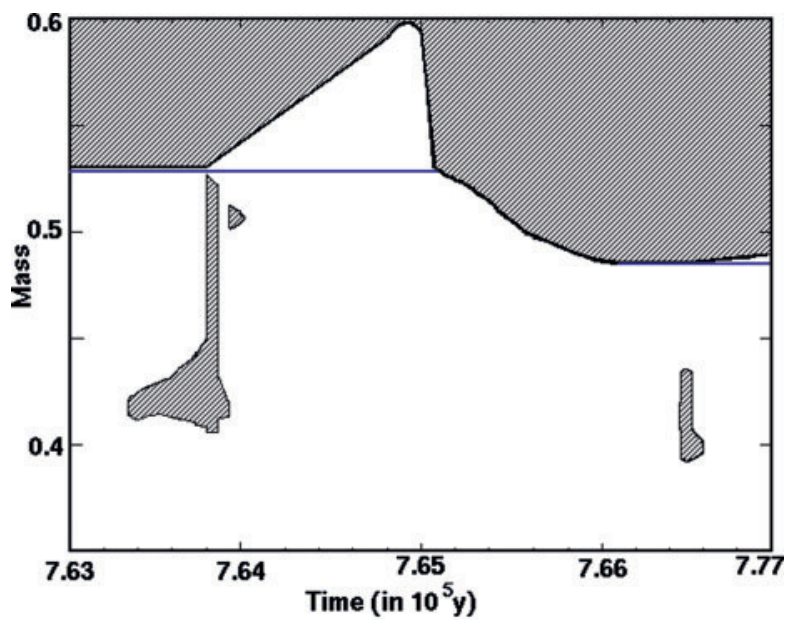

Figure 6 Close-up of the variation of the convective regions during the core flash for the $2 \mathrm{M}_{\odot}$ model with enhanced neutrino losses, model M2NL30, which results in a carbon star. Time is measured from $t=1.011 \times 10^{9}$ years.

Table 1. Abundances

\begin{tabular}{lcccc}
\hline Species $^{\mathrm{a}}$ & Range $^{\mathrm{b}}$ & M1.5NL1 & M1.5NL30 & M2NL30 \\
\hline$[\mathrm{Fe}]$ & $-0.40-0.19$ & 0 & 0 & 0 \\
{$[\mathrm{C}]$} & $0.12-0.77$ & -0.12 & 0.41 & 1.07 \\
{$[\mathrm{~N}]$} & $0.44-0.82$ & 0.29 & 0.28 & 0.33 \\
{$[\mathrm{O}]$} & $-0.53-0.04$ & 0.0008 & -0.009 & 0.0008 \\
$\mathrm{C} / \mathrm{O}$ & $0.9-3.3$ & 0.36 & 1.26 & 5.6 \\
$\mathrm{C} / \mathrm{N}$ & $1.5-9.3$ & 1.47 & 5.2 & 21 \\
{$[\mathrm{C}+\mathrm{N}+\mathrm{O}]$} & $0.1-0.4$ & 0 & 0.18 & 0.63 \\
${ }^{12} \mathrm{C} /{ }^{13} \mathrm{C}$ & $4-15$ & 27.5 & 99.8 & 459 \\
\hline
\end{tabular}

${ }^{a}$ Elemental abundances are given by number relative to the initial (solar) value: $[Y]=\log Y_{*}-\log Y_{\odot}$.

${ }^{\mathrm{b}}$ Range is the maximum and minimum observed values in the $\mathrm{R}$ stars studied by Dominy (1984).

became a carbon star, with $n(\mathrm{C}) / n(\mathrm{O})=5.6$; this larger value indicates that more carbon is dredged to the surface in this case.

\subsection{Nucleosynthesis}

The nucleosynthesis of most interest to us is the burning of helium into carbon, and its subsequent mixing to the surface. Nevertheless, we use a large network (74 species) to see if any other trace elements are produced which could be used as a probe or diagnostic of the proposed mixing. First we discuss the CNO species, which are the most important.

\subsubsection{CNO}

By far the most dominant effect of the altered evolution is that the dredge-up of primary carbon now produces an envelope that has $n(\mathrm{C})>n(\mathrm{O})$ so we form a carbon star as required. Quantitative results from the simulations are shown in Table 1. The carbon enhancement seen in the cases with increased neutrino loss fits reasonably well, certainly for the $1.5 \mathrm{M}_{\odot}$ model. The observations show 
Table 2. Approximate results of $\mathrm{CN}$ cycling

\begin{tabular}{lccccccccccc}
\hline Species & Range & $f=0.1$ & 0.2 & 0.3 & 0.4 & 0.5 & 0.6 & 0.7 & 0.8 & 0.9 & 0.96 \\
\hline M1.5NL30 & & & & & & & & & & & \\
C/O & $0.9-3.3$ & 1.13 & 1.01 & 0.88 & 0.76 & 0.63 & 0.50 & 0.38 & 0.25 & 0.13 & 0.05 \\
C/N & $1.5-9.3$ & 3.24 & 2.20 & 1.56 & 1.12 & 0.81 & 0.57 & 0.38 & 0.23 & 0.10 & 0.04 \\
${ }^{12} \mathrm{C} /{ }^{13} \mathrm{C}$ & $4-15$ & 90 & 80 & 70 & 60 & 50 & 40 & 30 & 20 & 10 & 4 \\
{$[\mathrm{~N}]$} & $0.44-0.82$ & 0.44 & 0.55 & 0.64 & 0.72 & 0.78 & 0.84 & 0.89 & 0.93 & 0.98 & 1.00 \\
M2NL30 & & & & & & & & & & & \\
C/O & $0.9-3.3$ & 5.06 & 4.50 & 3.94 & 3.37 & 2.81 & 2.25 & 1.69 & 1.12 & 0.56 & 0.23 \\
C/N & $1.5-9.3$ & 6.74 & 3.65 & 2.29 & 1.54 & 1.05 & 0.71 & 0.46 & 0.27 & 0.12 & 0.05 \\
${ }^{12} \mathrm{C} /{ }^{13} \mathrm{C}$ & $4-15$ & 411 & 364 & 317 & 270 & 224 & 178 & 133 & 89 & 44 \\
{$[\mathrm{~N}]$} & $0.44-0.82$ & 0.78 & 0.99 & 1.14 & 1.24 & 1.33 & 1.40 & 1.46 & 1.52 & 1.56 & 1.59 \\
\hline
\end{tabular}

substantial increases in $\mathrm{N}$ which we do not see in our models. The resulting $\mathrm{C} / \mathrm{O}$ values are in fair agreement, as is the sum $\mathrm{C}+\mathrm{N}+\mathrm{O}$. Indeed, it is perhaps this latter that is most important.

The observed R stars have enhanced $\mathrm{N}$, which is not seen in our models. Rather we have too much C. A simple solution to this is that the $\mathrm{C}$ may be processed into $\mathrm{N}$ by some form of additional mixing which can transport the enhanced $\mathrm{C}$ down to the $\mathrm{H}$ shell for processing, much as happens on the first giant branch. This would also contribute towards fixing our ${ }^{12} \mathrm{C} /{ }^{13} \mathrm{C}$ values which are also too large. All indications are that the carbon produced undergoes some $\mathrm{CN}$ cycling. We note that thermohaline mixing as discovered by Eggleton, Dearborn \& Lattanzio (2006) may be exactly what is required. Furthermore, a recent investigation by Cantiello et al. (2007) shows that this mixing can operate in low mass stars during the core helium burning stage. This may be the way the star processes much of the surface $\mathrm{C}$ into the observed $\mathrm{N}$.

We have made some approximate calculations to see if burning various amounts of the surface carbon can reproduce the observed abundances. The results are in Table 2. Here we assumed that a fraction $f$ of the surface carbon, after dredge-up, is processed by the CNO cycle into N. For simplicity we assume that all this processed $\mathrm{C}$ is ${ }^{12} \mathrm{C}$ and that it appears as ${ }^{14} \mathrm{~N}$. We approximate the small amount of ${ }^{13} \mathrm{C}$ produced by adding 0.001 times the final ${ }^{14} \mathrm{~N}$ abundance to the ${ }^{13} \mathrm{C}$ abundance resulting from our nucleosynthesis calculation. The results show that for reasonable values of $f$ we are able to reproduce all of the observed abundances except for the carbon isotopic ratio: the observed value is always smaller than our approximation. To match the observed value we must process essentially all of the additional $\mathrm{C}$ through $\mathrm{CN}$ cycling, which then results in overproduction of $\mathrm{N}$ and the decreased $\mathrm{C}$ content means that the star is no longer a $\mathrm{C}$ star! Similar problems exist with explaining the J stars. Note that one effect which works in our favour is the observed deep-mixing in stars on the first giant branch, which reduces the carbon isotope ratio below the value found at the first giant branch (e.g. Eggleton, Dearborn \& Lattanzio 2008). This was not included in our calculations and would have resulted in a lower ratio, perhaps closer to
14 or so, at the start of the merger rather than the value of 21 found in these calculations. This would go some way to reducing the discrepancy with the carbon isotope values.

Note that we have assumed $\mathrm{CN}$ cycling and not $\mathrm{ON}$ cycling. The fact that the $\mathrm{R}$ stars seem to show solar or slightly sub-solar oxygen may indicate that our simple approximation is not enough. A calculation including the $\mathrm{ON}$ cycle is required, but this would better take place within the context of a model for the deep mixing itself, and is deferred to subsequent work.

\subsubsection{Other Species}

We have included in our calculation some 74 species, including a small iron-peak network. With very few exceptions, the abundance changes seen in the potential $\mathrm{R}$ star models are negligible, being below $0.1 \mathrm{dex}$. The exceptions are the surface abundance of ${ }^{18} \mathrm{O}$, and ${ }^{22} \mathrm{Ne}$. We see a reasonably large increase in the heavy oxygen isotope by a factor of about 30 , from the totally negligible $X_{18} \simeq 2 \times 10^{-5}$ to the mostly negligible $X_{18} \simeq 5 \times 10^{-4}$. We do not expect this to be of diagnostic assistance however. For the unobservable ${ }^{22} \mathrm{Ne}$ the increase is a factor of 10-20, to the level of $X_{22} \simeq 0.001$. There is also a small, but temporary, increase in the radioactive isotope ${ }^{26} \mathrm{Al}$. Unfortunately, we see no changes in any species which may be used to test the hypothesis. Our best bet lies with the CNO species.

\section{Conclusion}

The R stars continue to resist theorists' attempts to determine their origin. The binary merger hypothesis seems to be the best candidate at present, but direct calculations of this stage are unavailable and we are forced to make small steps toward validating, or otherwise, this qualitative model. In this paper we have simulated the events that would follow a late ignition of the core flash. If this ignition occurs further from the centre than is normal, then we confirm that dredge-up of carbon may result. Our calculations show that a substantial fraction of this carbon must then be exposed to burning via the $\mathrm{CN}$ cycles (and possibly $\mathrm{ON}$ ). The observed low $\mathrm{C}$ isotope ratio remains a problem 
for the calculations shown here; the observed value indicates that essentially all of the added material has been burned to equilibrium via $\mathrm{CN}$ (and possibly $\mathrm{ON}$ ) cycling. Only in that case can we match the observed ${ }^{12} \mathrm{C} /{ }^{13} \mathrm{C}$ ratio. But then we burn too much $\mathrm{C}$ into $\mathrm{N}$, overproducing $\mathrm{N}$ and destroying so much $\mathrm{C}$ that the star is no longer a carbon star.

Further advances in understanding the $\mathrm{R}$ stars may require fully $3 \mathrm{D}$ hydrodynamical calculations of the merger event. Such work may be possible soon using the Duenuty code (e.g. Dearborn, Lattanzio \& Eggleton 2006).

\section{Acknowledgments}

This work was partially supported by the Australian Research Council and the Victorian Partnership for Advanced Computing. Thanks to Ross Church and Robert Izzard for discussions and comments. Thanks also to Lionel Siess for pointing out that a post-FDU merger event would assist with the carbon isotope ratios. J.L. also thanks the Australian wine industry for supporting this work, albeit indirectly. G.A. wishes to thank Carolyn Doherty, Simon Campbell and the other post grads in CSPA for their encouragement and support.

\section{References}

Catelan, M., de Freitas Pacheco, J. A. \& Horvath, J. E., 1996, ApJ, 461,231
Cantiello, M., Hoekstra, H., Langer, N. \& Poelarends, A. J. T., 2007, in AIPC 948, Unsolved Problems in Stellar Physics: A Conference in Honor of Douglas Gough, 73

Dearborn, D. S. P., Lattanzio, J. C. \& Eggleton, P. P., 2006, ApJ, 639,405

Despain, K. H., 1981, ApJ, 251, 639

Dominy, J. F., 1984, ApJS, 55, 27

Eggleton, P. P., Dearborn, D. S. P. \& Lattanzio, J. C., 2006, Sci, 314,1580

Eggleton, P. P., Dearborn, D. S. P. \& Lattanzio, J. C., 2008, ApJ, 677,581

Frost, C. A. \& Lattanzio, J. C., 1996, ApJ, 473, 383

Fujimoto, M. Y., Iben, I., Jr. \& Hollowell, D., 1990, ApJ, 349, 580

Fujimoto, M. Y., Ikeda, Y. \& Iben, I., Jr., 2000, ApJ, 529, L25

Hollowell, D., Iben, I., Jr. \& Fujimoto, M. Y., 1990, ApJ, 351, 245

Izzard, R. G., Jeffery, C. S. \& Lattanzio, J. C., 2007, A \& A, 470, 661

Komiya, Y., Suda, T., Minaguchi, H., Shigeyama, T., Aoki, W. \& Fujimoto, M. Y., 2007, ApJ, 658, 367

Lattanzio, J. C., Frost, C. A., Cannon, R. C. \& Wood, P. R., 1996, MmSAI, 67, 729

Lugaro, M., Ugalde, C., Karakas, A. I., Gorres, J., Wiescher, M., Lattanzio, J. C. \& Cannon, R. C., 2004, ApJ, 615, 934

McClure, R. D., 1997, PASP, 109, 256

Paczyński, B. \& Tremaine, S. D., 1977, ApJ, 216, 57

Picard, I., Chieffi, A., Limongi, M., Pisanti, O., Miele, G., Mangano, G. \& Imbriani, G., 2004, ApJ, 609, 1035

Schlattl, H., Cassisi, S., Salaris, M. \& Weiss, A., 2001, ApJ, 559, 1082

Schlattl, H., Salaris, M., Cassisi, S. \& Weiss, A., 2002, A \& A, 395, 77 